\title{
ON DEPENDENT ELEMENTS IN RINGS
}

\author{
JOSO VUKMAN and IRENA KOSI-ULBL
}

Received 18 November 2003

Let $R$ be an associative ring. An element $a \in R$ is said to be dependent on a mapping $F$ : $R \rightarrow R$ in case $F(x) a=a x$ holds for all $x \in R$. In this paper, elements dependent on certain mappings on prime and semiprime rings are investigated. We prove, for example, that in case we have a semiprime ring $R$, there are no nonzero elements which are dependent on the mapping $\alpha+\beta$, where $\alpha$ and $\beta$ are automorphisms of $R$.

2000 Mathematics Subject Classification: 16E99, 16W10, 13N15, 08 A35.

This research has been motivated by the work of Laradji and Thaheem [11]. Throughout, $R$ will represent an associative ring with center $Z(R)$. As usual the commutator $x y-y x$ will be denoted by $[x, y]$. We will use basic commutator identities $[x y, z]=$ $[x, z] y+x[y, z]$ and $[x, y z]=[x, y] z+y[x, z]$. Recall that a ring $R$ is prime if $a R b=$ (0) implies that $a=0$ or $b=0$, and is semiprime if $a R a=(0)$ implies $a=0$. An additive mapping $x \mapsto x^{*}$ on a ring $R$ is called involution in case $(x y)^{*}=y^{*} x^{*}$ and $x^{* *}=x$ hold for all $x, y \in R$. A ring equipped with an involution is called a ring with involution or ${ }^{*}$-ring. An additive mapping $D: R \rightarrow R$ is called a derivation in case $D(x y)=D(x) y+x D(y)$ holds for all pairs $x, y \in R$. A derivation $D$ is inner in case there exists $a \in R$ such that $D(x)=[a, x]$ holds for all $x \in R$. An additive mapping $T: R \rightarrow R$ is called a left centralizer in case $T(x y)=T(x) y$ is fulfilled for all pairs $x, y \in R$. This concept appears naturally in $C^{*}$-algebras. In ring theory it is more common to work with module homomorphisms. Ring theorists would simply write that $T: R_{R} \rightarrow R_{R}$ is a homomorphism of a right $R$-module $R$ into itself. For any fixed element $a \in R$, the mapping $T(x)=a x, x \in R$, is a left centralizer. In case $R$ has the identity element $T: R \rightarrow R$ is a left centralizer if and only if $T$ is of the form $T(x)=a x$, $x \in R$, where $a \in R$ is a fixed element. For a semiprime ring $R$, a mapping $T: R \rightarrow R$ is a left centralizer if and only if $T(x)=q x$ holds for all $x \in R$, where $q$ is an element of Martindale right ring of quotients $Q_{r}$ (see [1, Chapter 2]). An additive mapping $T: R \rightarrow R$ is said to be a right centralizer in case $T(x y)=x T(y)$ holds for all pairs $x, y \in R$. In case $R$ has the identity element $T: R \rightarrow R$ is both left and right centralizer if and only if $T(x)=a x, x \in R$, where $a \in Z(R)$ is a fixed element. In case $R$ is a semiprime ring with extended centroid $C$ a mapping $T: R \rightarrow R$ is both left and right centralizer in case $T$ is of the form $T(x)=\lambda x, x \in R$, where $\lambda \in C$ is a fixed element (see [1, Theorem 2.3.2]). For results concerning centralizers on prime and semiprime rings, we refer to [18, 19, 20, 21, 22, 23, 24]. Following [11] an element $a \in R$ is said to be an element dependent on a mapping $F: R \rightarrow R$ if $F(x) a=a x$ holds for all $x \in R$. A mapping $F: R \rightarrow R$ is called a free action in case zero is the only element dependent 
on $F$. It is easy to see that in semiprime rings there are no nonzero nilpotent dependent elements (see [11]). This fact will be used throughout the paper without specific references. Dependent elements were implicitly used by Kallman [10] to extend the notion of free action of automorphisms of abelian von Neumann algebras of Murray and von Neumann [14, 17]. They were later on introduced by Choda et al. [8]. Several other authors have studied dependent elements in operator algebras (see [6, 7]). A brief account of dependent elements in $W^{*}$-algebras has been also appeared in the book of Strătilă [16]. The purpose of this paper is to investigate dependent elements of some mappings related to derivations and automorphisms on prime and semiprime rings.

We will need the following two lemmas.

LEMMA 1 (see [2, Lemma 4]). Let $R$ be a 2-torsion-free semiprime ring and let $a, b \in R$. If, for all $x \in R$, the relation $a \times b+b \times a=0$ holds, then $a \times b=b \times a=0$ is fulfilled for all $x \in R$.

LEMMA 2 (see [12, Theorem 1]). Let $R$ be a prime ring with extended centroid $C$ and let $a, b \in R$ be such that $a \times b=b \times a$ holds for all $x \in R$. If $a \neq 0$, then there exists $\lambda \in C$ such that $b=\lambda a$.

Our first result has been motivated by Posner's first theorem [15] which states that the compositum of two nonzero derivations on a 2-torsion-free prime ring cannot be a derivation.

THEOREM 3. Let $R$ be a semiprime ring and let $D$ and $G$ be derivations of $R$ into itself. In this case the mapping $x \mapsto D^{2}(x)+G(x)$ is a free action.

Proof. We have the relation

$$
F(x) a=a x, \quad x \in R,
$$

where $F(x)$ stands for $D^{2}(x)+G(x)$. A routine calculation shows that the relation

$$
F(x y)=F(x) y+x F(y)+2 D(x) D(y)
$$

holds for all pairs $x, y \in R$. Putting $x a$ for $x$ in (1) and using (2) we obtain $F(x) a^{2}+$ $x F(a) a+2 D(x) D(a) a=a x a, x \in R$, which reduces because of (1) to

$$
2 D(x) D(a) a+x a^{2}=0, \quad x \in R .
$$

Putting, in the above relation, $y x$ for $x$ and applying (3) we obtain $2 D(y) x D(a) a=0$, $x, y \in R$, whence it follows, putting $D(x)$ for $x$, that

$$
2 D(y) D(x) D(a) a=0, \quad x, y \in R .
$$

Multiplying relation (3) from the left by $D(y)$ and applying the above relation we obtain $D(y) x a^{2}=0, x, y \in R$, which gives, for $x=D(a)$ and $y=a$,

$$
D(a)^{2} a^{2}=0 .
$$


Multiplying relation (3) from the right by $a$, putting $x=a$ in (3), and applying the above relation we obtain $a^{4}=0$, which means that also $a=0$. The proof of the theorem is complete.

For our next result, we need the concept of the so-called generalized derivations introduced by Brešar in [3]. An additive mapping $F: R \rightarrow R$, where $R$ is an arbitrary ring, is called a generalized derivation in case $F(x y)=F(x) y+x D(y)$ holds for all pairs $x, y \in R$, where $D: R \rightarrow R$ is a derivation. It is easy to see that $F$ is a generalized derivation if and only if $F$ is of the form $F=D+T$, where $D$ is a derivation and $T$ a left centralizer. For some results concerning generalized derivations, we refer the reader to [9].

THEOREM 4. Let $F: R \rightarrow R$ be a generalized derivation, where $R$ is a semiprime ring, and let $a \in R$ be an element dependent on $F$. In this case $a \in Z(R)$.

Proof. We have the relation

$$
F(x) a=a x, \quad x \in R .
$$

Let $x$ be $x y$ in the above relation. Then we have

$$
(F(x) y+x D(y)) a=a x y, \quad x, y \in R .
$$

Using the fact that $F$ can be written in the form $F=D+T$, where $T$ is a left centralizer, we can replace $D(y) a$ by $F(y) a-T(y) a$ in (7), which gives, because of (6),

$$
F(x) y a+[x, a] y-x T(y) a=0, \quad x, y \in R .
$$

Let $y$ be $y F(x)$ in (8). We have

$$
F(x) y F(x) a+[x, a] y F(x)-x T(y) F(x) a=0, \quad x, y \in R,
$$

which reduces, according to (6), to

$$
F(x) y a x+[x, a] y F(x)-x T(y) a x=0, \quad x, y \in R .
$$

Right multiplication of (8) by $x$ gives

$$
F(x) y a x+[x, a] y x-x T(y) a x=0, \quad x, y \in R .
$$

Subtracting (11) from (10) we arrive at

$$
[x, a] y(F(x)-x)=0, \quad x, y \in R \text {. }
$$

Right multiplication of the above relation by a gives, because of $(6),[x, a] y[x, a]=0$, $x, y \in R$, whence it follows that $[x, a]=0, x \in R$. The proof of the theorem is complete.

COROLlARY 5. Let $R$ be a semiprime ring and let $a, b \in R$ be fixed elements. Suppose that $c \in R$ is an element dependent on the mapping $x \mapsto a x+x b$. In this case $c \in Z(R)$. 
Proof. A special case of Theorem 4, since it is easy to see that the mapping $x \mapsto$ $a x+x b$ is a generalized derivation.

In the theory of operator algebras the mappings $x \mapsto a x+x b$, which we met in the above corollary, are considered as an important class of the so-called elementary operators (i.e., mappings of the form $x \mapsto \sum_{i=1}^{n} a_{i} x b_{i}$ ). We refer the reader to [13] for a good account of this theory.

THEOREM 6. Let $R$ be a noncommutative prime ring with extended centroid $C$ and let $a, b \in R$ be fixed elements. Suppose that $c \in R$ is an element dependent on the mapping $x \mapsto a x b$. In this case the following statements hold:

(1) $b c \in Z(R)$;

(2) $a b c=c$;

(3) $c=\lambda a$ for some $\lambda \in C$.

Proof. We will assume that $a \neq 0$ and $b \neq 0$ since there is nothing to prove in case $a=0$ or $b=0$. We have

$$
(a x b) c=c x, \quad x \in R
$$

Let $x$ be $x y$ in (13). Then

$$
(a x y b) c=c x y, \quad x, y \in R
$$

According to (13) one can replace $c x$ by $(a x b) c$ in the above relation. Then we have

$$
a x[b c, y]=0, \quad x, y \in R,
$$

which gives $b c \in Z(R)$, which makes it possible to rewrite relation (13) in the form

$$
(a b c-c) x=0, \quad x \in R
$$

whence it follows that

$$
a b c=c
$$

Putting $x a$ for $x$ in relation (13) we obtain, because of (17),

$$
a x c=c x a, \quad x \in R,
$$

whence it follows, according to Lemma 2, that $c=\lambda a$ for some $\lambda \in C$. The proof of the theorem is complete.

COROLLARY 7. Let $R$ be a noncommutative prime ring with the identity element and extended centroid $C$ and let $\alpha(x)=a x a^{-1}, x \in R$, be an inner automorphism of $R$. An element $b \in R$ is an element dependent on $\alpha$ if and only if $b=\lambda a$ for some $\lambda \in C$.

Proof. According to Theorem 6 any element dependent on $\alpha$ is of the form $\lambda a$ for some $\lambda \in C$. It is trivial to see that any element of the form $\lambda a$, where $\lambda \in C$, is an element dependent on $\alpha$. 
We proceed to our next result.

THEOREM 8. Let $R$ be a noncommutative 2-torsion-free prime ring and let $a, b \in R$ be fixed elements. Suppose that $c \in R$ is an element dependent on the mapping $x \mapsto$ $a \times b+b x a$. In this case the following statements hold:

(1) $a c \in Z(R)$ and $b c \in Z(R)$;

(2) $(a b+b a) c=c$;

(3) $c^{2} \in Z(R)$.

Proof. Similarly, as in the proof of Theorem 6 , we will assume that $a \neq 0$ and $b \neq 0$. We have the relation

$$
(a x b+b \times a) c=c x, \quad x \in R .
$$

Let $x$ be $x y$ in the above relation. Then we have

$$
(a x y b+b x y a) c=c x y, \quad x, y \in R .
$$

Right multiplication of relation (19) by $y$ gives

$$
(a x b+b x a) c y=c x y, \quad x, y \in R .
$$

Subtracting (21) from (20) we arrive at

$$
a x[y, b c]+b x[y, a c]=0, \quad x, y \in R .
$$

Putting $c x$ for $x$ in the above relation we arrive at

$$
a c x[y, b c]+b c x[y, a c]=0, \quad x, y \in R .
$$

Now, multiplying the above relation first from the left by $y$, then putting $y x$ for $x$ in (23), and finally subtracting the relations so obtained from one another, we arrive at

$$
[y, a c] x[y, b c]+[y, b c] x[y, a c]=0, \quad x, y \in R .
$$

Suppose that $a c \notin Z(R)$. In this case we have $[y, a c] \neq 0$ for some $y \in R$. Then it follows from relation (24) and Lemma 1 that $[y, b c]=0$, which reduces relation (22) to $b x[y, a c]=0, x, y \in R$, which means (recall that $b$ is different from zero) that $[y, a c]=0$, contrary to the assumption. We have therefore $a c \in Z(R)$. Now relation (22) reduces to $a x[y, b c]=0, x, y \in R$, whence it follows that $b c \in Z(R)$. Since $a c$ and $b c$ are in $Z(R)$, one can write relation (19) in the form $((a b+b a) c-c) x=0, x \in R$, which gives

$$
(a b+b a) c=c .
$$

Putting $x=c$ in relation (19) we obtain

$$
2(a c)(b c)=c^{2} .
$$

Since $a c$ and $b c$ are both in $Z(R)$ it follows from the above relation that $c^{2} \in Z(R)$. The proof of the theorem is complete. 
THEOREM 9. Let $R$ be a noncommutative 2-torsion-free prime ring with extended centroid $C$ and let $a, b \in R$ be fixed elements. In this case the mapping $x \mapsto a x b-b x a$ is a free action.

Proof. Again we assume that $a \neq 0$ and $b \neq 0$. Besides, we will also assume that $a$ and $b$ are $C$-independent, otherwise the mapping $x \mapsto a x b-b x a$ would be zero. We have the relation

$$
(a x b-b x a) c=c x, \quad x \in R .
$$

Let $x$ be $x y$ in the above relation. Then we have

$$
(a x y b-b x y a) c=c x y, \quad x, y \in R \text {. }
$$

Right multiplication of relation (27) by $y$ gives

$$
(a x b-b x a) c y=c x y, \quad x, y \in R
$$

Subtracting (29) from (28) we arrive at

$$
a x[y, b c]-b x[y, a c]=0, \quad x, y \in R .
$$

Putting $c x$ for $x$ in the above relation we arrive at

$$
a c x[y, b c]-b c x[y, a c]=0, \quad x, y \in R .
$$

Now, multiplying first the above relation from the left by $y$, then putting $y x$ for $x$ in (31), and finally subtracting the relations so obtained from one another, we arrive at

$$
[y, a c] x[y, b c]-[y, b c] x[y, a c]=0, \quad x, y \in R .
$$

Suppose that $a c \notin Z(R)$. In this case there exists $y \in R$ such that $[y, a c] \neq 0$. Now it follows from the above relation and from Lemma 2 that

$$
[y, b c]=\lambda_{y}[y, a c]
$$

holds for some $\lambda_{y} \in C$. According to (33) one can replace $[y, b c]$ by $\lambda_{y}[y, a c]$ in (30), which gives

$$
\left(b-\lambda_{y} a\right) x[y, a c]=0, \quad x \in R .
$$

Since $[y, a c] \neq 0$ it follows from the above relation that $b=\lambda_{y} a$, contrary to the assumption that $a$ and $b$ are $C$-independent. We have therefore proved that $a c \in Z(R)$. Using this fact relation (30) reduces to

$$
a x[y, b c]=0, \quad x, y \in R,
$$

whence it follows (recall that $a \neq 0$ ) that $b c \in Z(R)$. Since $a c$ and $b c$ are both in $Z(R)$, one can rewrite relation (27) in the form $((a b-b a) c-c) x=0, x \in R$, which gives

$$
(a b-b a) c=c .
$$


Putting $x=c$ in relation (27) and using the fact that $b c$ is in $Z(R)$, we obtain

$$
a[b, c] c=-c^{2} .
$$

From relation (36) one obtains, using the fact that $a c \in Z(R)$,

$$
a[b, c]=c .
$$

Right multiplication of the above relation by $c$ gives

$$
a[b, c] c=c^{2} .
$$

Comparing relations (37) and (39) one obtains $c^{2}=0$, since $R$ is 2-torsion-free. Now it follows that $c=0$, which completes the proof of the theorem.

THEOREM 10. Let $R$ be a semiprime ring and let $\alpha$ and $\beta$ be automorphisms of $R$. In this case the mapping $\alpha+\beta$ is a free action.

Proof. We have the relation

$$
(\alpha(x)+\beta(x)) a=a x, \quad x \in R .
$$

Let $x$ be $x y$ in the above relation. Then

$$
(\alpha(x) \alpha(y)+\beta(x) \beta(y)) a=a x y, \quad x, y \in R .
$$

Replacing first $a x$ by $(\alpha(x)+\beta(x)) a$ in the above relation and then $a y$ by $(\alpha(y)+$ $\beta(y)) a$, we arrive at

$$
(\alpha(x) \alpha(y)+\beta(x) \beta(y)) a=(\alpha(x)+\beta(x))(\alpha(y)+\beta(y)) a, \quad x, y \in R,
$$

which reduces to

$$
\alpha(x) \beta(y) a+\beta(x) \alpha(y) a=0, \quad x, y \in R .
$$

The substitution $z x$ for $x$ in the above relation gives

$$
\alpha(z) \alpha(x) \beta(y) a+\beta(z) \beta(x) \alpha(y) a=0, \quad x, y, z \in R .
$$

Left multiplication of (43) by $\alpha(z)$ gives

$$
\alpha(z) \alpha(x) \beta(y) a+\alpha(z) \beta(x) \alpha(y) a=0, \quad x, y, z \in R .
$$

Subtracting (44) from (45), we arrive at $(\alpha(z)-\beta(z)) \beta(x) \alpha(y) a=0, x, y, z \in R$. We therefore have

$$
(\alpha(z)-\beta(z)) x y a=0, \quad x, y, z \in R .
$$

Putting $x=a$ and $y(\alpha(z)-\beta(z))$ for $y$ in the above relation, we obtain $(\alpha(z)-$ $\beta(z)) a x(\alpha(z)-\beta(z)) a=0, x, z \in R$, whence it follows that

$$
\alpha(z) a=\beta(z) a, \quad z \in R .
$$


According to (47) one can replace $\beta(y) a$ by $\alpha(y) a$ in (43), which gives $(\alpha(x)+$ $\beta(x)) \alpha(y) a=0, x, y \in R$. We therefore have

$$
(\alpha(x)+\beta(x)) y a=0, \quad x, y \in R .
$$

Putting $y=a$ in the above relation and replacing $(\alpha(x)+\beta(x)) a$ by $a x$, we obtain $a x a=0, x \in R$, which gives $a=0$. The proof of the theorem is complete.

The following question arises: what can be proved in case we have $\alpha-\beta$ instead of $\alpha+\beta$ in the above theorem? The mapping $\alpha-\beta$ is a special case of the so-called $(\alpha, \beta)$-derivations. An additive mapping $D: R \rightarrow R$, where $R$ is an arbitrary ring, is an $(\alpha, \beta)$-derivation if $D(x y)=D(x) \alpha(y)+\beta(x) D(y)$ holds for all pairs $x, y \in R$, where $\alpha$ and $\beta$ are automorphisms of $R$. For results concerning $(\alpha, \beta)$-derivations, we refer the reader to $[4,5]$.

THEOREM 11. Let $R$ be a semiprime ring and let $D: R \rightarrow R$ be an $(\alpha, \beta)$-derivation. In this case $D$ is a free action.

Proof. We have the relation

$$
D(x) a=a x, \quad x \in R .
$$

Putting $x y$ for $x$ in the above relation we obtain

$$
D(x) \alpha(y) a+\beta(x) D(y) a=a x y, \quad x, y \in R .
$$

According to (49) one can replace $D(y) a$ by ay in the above relation, which gives

$$
D(x) \alpha(y) a+(\beta(x) a-a x) y=0, \quad x, y \in R .
$$

Putting $y z$ for $y$ in (51) we obtain

$$
D(x) \alpha(y) \alpha(z) a+(\beta(x) a-a x) y z=0, \quad x, y, z \in R .
$$

On the other hand, right multiplication of (51) by $z$ gives

$$
D(x) \alpha(y) a z+(\beta(x) a-a x) y z=0, \quad x, y, z \in R .
$$

Subtracting (53) from (52) we obtain $D(x) \alpha(y)(\alpha(z) a-a z)=0, x, y, z \in R$. In other words, we have

$$
D(x) y(\alpha(z) a-a z)=0, \quad x, y, z \in R .
$$

The substitution ay for $y$ in the above relation gives, because of (49),

$$
\operatorname{axy}(\alpha(z) a-a z)=0, \quad x, y, z \in R .
$$

Putting $z x$ for $x$ in the above relation we obtain

$$
\operatorname{azxy}(\alpha(z) a-a z)=0, \quad x, y, z \in R
$$


Left multiplication of (55) by $\alpha(z)$ gives

$$
\alpha(z) a x y(\alpha(z) a-a z)=0, \quad x, y, z \in R .
$$

Subtracting (56) from (57) and multiplying the relation so obtained from the righthand side by $x$, we arrive at

$$
(\alpha(z) a-a z) x y(\alpha(z) a-a z) x=0, \quad x, y, z \in R,
$$

which gives first

$$
(\alpha(z) a-a z) x=0, \quad x, z \in R
$$

and then

$$
\alpha(z) a-a z=0, \quad z \in R
$$

Putting $D(x) a$ instead of $a x$ in (50), and $a y$ for $D(y) a$, we obtain $D(x)(\alpha(y) a-$ $a y)+\beta(x) a y=0, x, y \in R$, which reduces because of (60) to $\beta(x) a y=0, x, y \in R$, whence it follows that $a=0$. The proof of the theorem is complete.

COROLLARY 12. Let $R$ be a semiprime ring and let $\alpha$ and $\beta$ be automorphisms of $R$. In this case the mappings $\alpha-\beta$ and $a \alpha-\beta a$, where $a \in R$ is a fixed element, are free actions on $R$.

Proof. According to Theorem 11 there is nothing to prove, since the mappings $\alpha-\beta$ and $a \alpha-\beta a$ are $(\alpha, \beta)$-derivations.

COROLlary 13. Let $R$ be a semiprime ring, let $D: R \rightarrow R$ be a derivation, and let $\alpha$ be an automorphism of $R$. In this case the mappings $x \mapsto D(\alpha(x)), x \mapsto \alpha(D(x))$, $x \mapsto D(\alpha(x))+\alpha(D(x))$, and $x \mapsto D(\alpha(x))-\alpha(D(x))$ are free actions.

Proof. A special case of Theorem 11, since all mappings are $(\alpha, \alpha)$-derivations.

For our next result we need the following lemma.

LEMMA 14 (see [24, Lemma 1.3]). Let $R$ be a semiprime ring and let $a \in R$ be a fixed element. If $a[x, y]=0$ holds for all pairs $x, y \in R$, then there exists an ideal I of $R$ such that $a \in I \subset Z(R)$.

Proposition 15. Let $R$ be a semiprime ring and let $\alpha: R \rightarrow R$ be an antiautomorphism. Suppose $a \in R$ is an element dependent on $\alpha$. In this case there exists an ideal I of $R$ such that $a \in I \subset Z(R)$. In case $R$ is a prime ring, then either $\alpha$ is a free action or $\alpha$ is the identity mapping and $R$ is commutative.

Proof. We have the relation

$$
\alpha(x) a=a x, \quad x \in R .
$$


Putting $x y$ for $y$ in (61) and using (61) we obtain

$$
a x y=\alpha(x y) a=\alpha(y) \alpha(x) a=\alpha(y) a x=a y x .
$$

We therefore have

$$
a[x, y]=0, \quad x, y \in R
$$

From (63) and Lemma 14 it follows that there exists an ideal $I$ of $R$ such that $a \in$ $I \subset Z(R)$, which completes the first part of the proof. The fact that $a \in Z(R)$ makes it possible to rewrite relation (61) in the form $(\alpha(x)-x) a=0, x \in R$, whence it follows that

$$
(\alpha(x)-x) y a=0, \quad x, y \in R
$$

In case $R$ is a prime ring it follows from the above relation that either $a=0$ or $\alpha(x)=x$ for all $x \in R$, which completes the proof of the theorem.

Proposition 16. Let $R$ be a semiprime *-ring. Suppose that $a \in R$ is dependent on the involution. In this case there exists an ideal $I$ of $R$ such that $a \in I \subset Z(R)$ and $a^{*}=a$. In case $R$ is a prime ring, then either the involution is a free action or the involution is the identity mapping and $R$ is commutative.

Proof. Since all the assumptions of Proposition 15 are fulfilled, it remains to prove that $a^{*}=a$. Putting

$$
x^{*} a=a x, \quad x \in R
$$

and $x=a$ in the relation we obtain $a^{2}=a^{*} a$, which can be written in the form

$$
\left(a-a^{*}\right) a=0
$$

From the above relation we obtain, using the fact that $a \in Z(R)$,

$$
0=\left(a\left(a-a^{*}\right)\right)^{*}=\left(a-a^{*}\right) a^{*}
$$

Thus we have

$$
\left(a-a^{*}\right) a^{*}=0 .
$$

Right multiplication of (66) by $x$ gives

$$
\left(a-a^{*}\right) x a=0, \quad x \in R,
$$

since $a \in Z(R)$. Similarly, from (68) one obtains (note that also $a^{*} \in Z(R)$ )

$$
\left(a-a^{*}\right) x a^{*}=0, \quad x \in R .
$$


Subtracting (70) from (69), we obtain

$$
\left(a-a^{*}\right) x\left(a-a^{*}\right)=0, \quad x \in R,
$$

whence it follows that $a^{*}=a$, which completes the proof.

THEOREM 17. Let $R$ be a semiprime ring and let $\alpha$ be an antiautomorphism of $R$. In this case the mapping $x \mapsto \alpha(x)+x$ is a free action.

Proof. We have $(\alpha(x)+x) a=a x, x \in R$, which can be written in the form

$$
\alpha(x) a=D(x), \quad x \in R,
$$

where $D(x)$ stands for $[a, x]$. Putting $x y$ for $x$ in the above relation we obtain

$$
\alpha(y) \alpha(x) a=D(x) y+x D(y), \quad x, y \in R .
$$

According to (72) one can replace $\alpha(x) a$ by $D(x)$ in the above relation, which gives

$$
\alpha(y) D(x)=D(x) y+x D(y), \quad x, y \in R .
$$

Putting $x=a$ in the above relation (note that $D(a)=0$ ) one obtains

$$
a D(y)=0, \quad y \in R
$$

According to (75), left multiplication of relation (72) by $a$ reduces it to $a \alpha(x) a=0$, $x \in R$, whence it follows that $a=0$ by semiprimeness of $R$, which completes the proof.

COROLlARY 18. Let $R$ be a semiprime ${ }^{*}$-ring. The mapping $x \mapsto x^{*}+x$ is a free action.

ACKNOWLEDGMENT. This work was supported by the Research Council of Slovenia.

\section{REFERENCES}

[1] K. I. Beidar, W. S. Martindale III, and A. V. Mikhalev, Rings with Generalized Identities, Monographs and Textbooks in Pure and Applied Mathematics, vol. 196, Marcel Dekker, New York, 1996.

[2] M. Brešar, Jordan derivations on semiprime rings, Proc. Amer. Math. Soc. 104 (1988), no. 4, 1003-1006.

[3] _ On the distance of the composition of two derivations to the generalized derivations, Glasgow Math. J. 33 (1991), no. 1, 89-93.

[4] On the compositions of $(\alpha, \beta)$-derivations of rings, and applications to von Neumann algebras, Acta Sci. Math. (Szeged) 56 (1992), no. 3-4, 369-375.

[5] J.-C. Chang, $(\alpha, \beta)$-derivation of prime rings having power central values, Bull. Inst. Math. Acad. Sinica 23 (1995), no. 4, 295-303.

[6] H. Choda, On freely acting automorphisms of operator algebras, Keio Math. Sem. Rep. 26 (1974), 1-21.

[7] H. Choda and Y. Watatani, Subfreely acting automorphisms of operator algebras, Math. Japon. 26 (1981), no. 2, 223-232.

[8] M. Choda, I. Kasahara, and R. Nakamoto, Dependent elements of an automorphism of a $C^{*}$-algebra, Proc. Japan Acad. 48 (1972), 561-565. 
[9] B. Hvala, Generalized derivations in rings, Comm. Algebra 26 (1998), no. 4, 1147-1166.

[10] R. R. Kallman, A generalization of free action, Duke Math. J. 36 (1969), 781-789.

[11] A. Laradji and A. B. Thaheem, On dependent elements in semiprime rings, Math. Japon. 47 (1998), no. 1, 29-31.

[12] W. S. Martindale III, Prime rings satisfying a generalized polynomial identity, J. Algebra 12 (1969), 576-584.

[13] M. Mathieu (ed.), Elementary Operators \& Applications, Proceedings of the International Workshop, World Scientific Publishing, New Jersey, 1992.

[14] F. J. Murray and J. von Neumann, On rings of operators, Ann. of Math. (2) 37 (1936), no. 1, 116-229.

[15] E. C. Posner, Derivations in prime rings, Proc. Amer. Math. Soc. 8 (1957), 1093-1100.

[16] Ş. Strătilă, Modular Theory in Operator Algebras, Editura Academiei Republicii Socialiste România, Bucharest; Abacus Press, Kent, 1981.

[17] J. von Neumann, On rings of operators. III, Ann. of Math. (2) 41 (1940), 94-161.

[18] J. Vukman, Centralizers on prime and semiprime rings, Comment. Math. Univ. Carolin. 38 (1997), no. 2, 231-240.

[19] _ An identity related to centralizers in semiprime rings, Comment. Math. Univ. Carolin. 40 (1999), no. 3, 447-456.

[20] _ Centralizers on semiprime rings, Comment. Math. Univ. Carolin. 42 (2001), no. 2, 237-245.

[21] J. Vukman and I. Kosi-Ulbl, On centralizers in rings and algebras with involution, in preparation.

[22] _ _ An equation related to centralizers in semiprime rings, Glas. Mat. Ser. III 38(58) (2003), no. 2, 253-261.

[23] _ On centralizers of semiprime rings, Aequationes Math. 66 (2003), no. 3, 277-283.

[24] B. Zalar, On centralizers of semiprime rings, Comment. Math. Univ. Carolin. 32 (1991), no. 4, 609-614.

Joso Vukman: Department of Mathematics, University of Maribor, Koroška cesta 160, 2000 Maribor, Slovenia

E-mail address: joso.vukman@uni-mb.si

Irena Kosi-Ulbl: Department of Mathematics, University of Maribor, Koroška cesta 160, 2000 Maribor, Slovenia

E-mail address: irena.kosi@uni-mb.si 


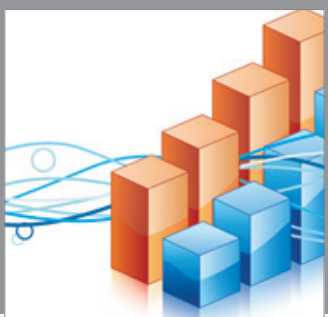

Advances in

Operations Research

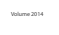

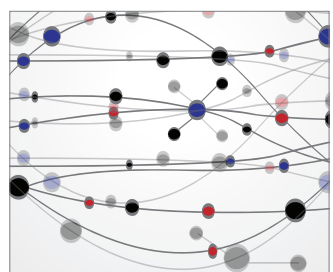

\section{The Scientific} World Journal
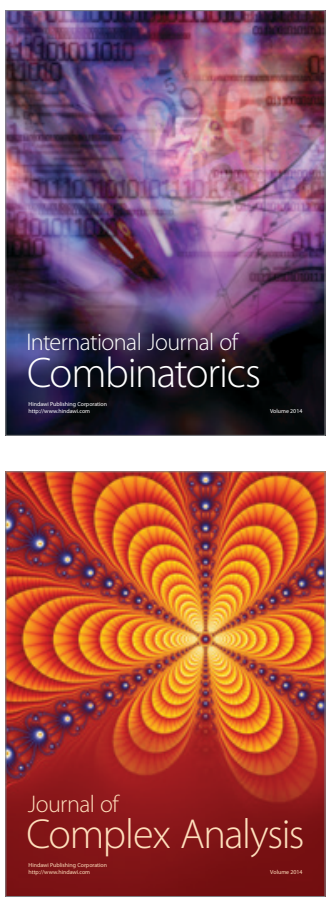

International Journal of

Mathematics and

Mathematical

Sciences
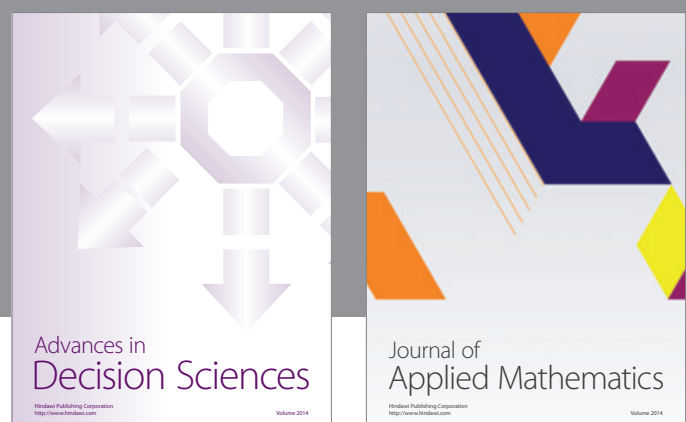

Journal of

Applied Mathematics
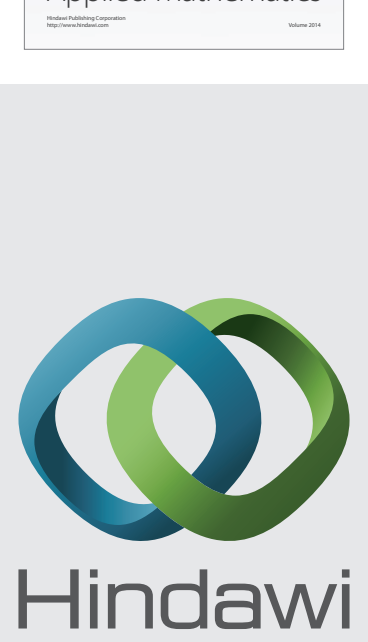

Submit your manuscripts at http://www.hindawi.com
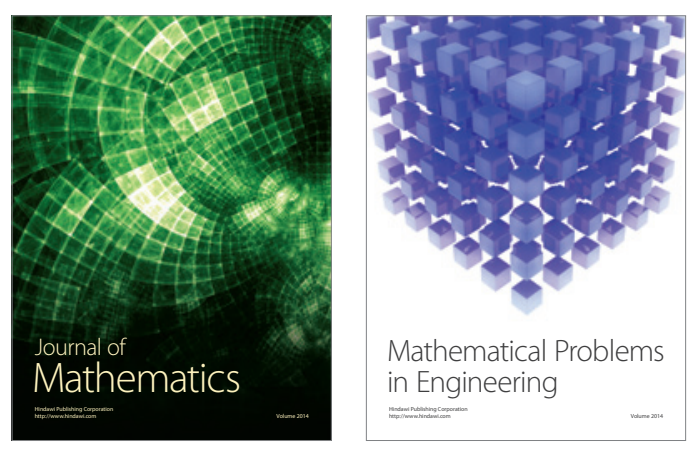

Mathematical Problems in Engineering
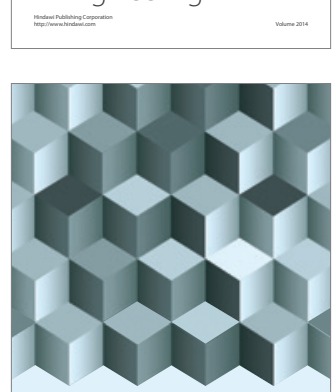

Journal of

Function Spaces
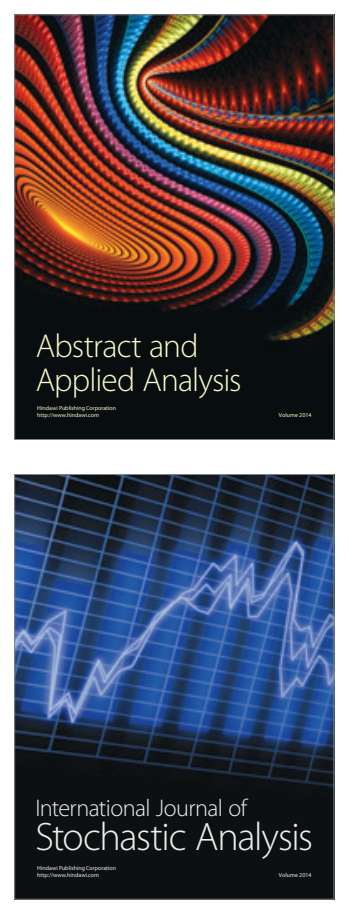

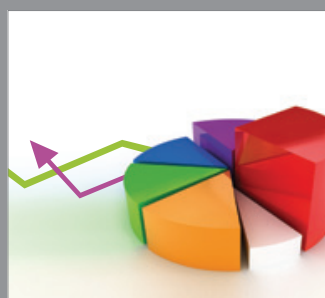

ournal of

Probability and Statistics

Promensencen
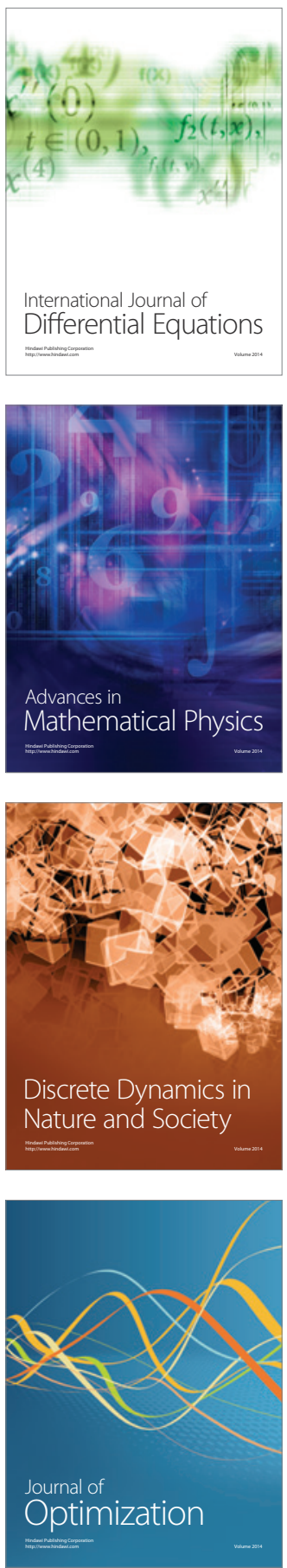